\title{
N87-29918
}

\section{A VIEW FROM THE AIAA: INTRODUCTION OF NEW ENERG STORAGE TECHNOLOGY INTO ORBITAL PROGRAMS}

\author{
Charles Badcock \\ The Aerospace Corporation \\ Los Angeles, California 90009
}

\begin{abstract}
The development of new energy storage technology must be heavily weighted toward the application. The requirements for transitioning low risk technology into operational space vehicles must remain the central theme even at the preliminary development stages by the development of efforts to define operational issues and verify the reliability of the system. Failure to follow a complete plan that results in a flight qualified unit may lead to an orphan technology. Development efforts must be directed toward a stable development where changes in design are evolutionary and end items are equivalent to flight units so that life and qualification testing can be used as a vehicle to demonstrate the acceptability of the
\end{abstract} technology.

\section{INTRODUCTION}

The American Institute of Aeronautics and Astronautics promotes the development and application of aircraft and aerospace technologies. We participate in the formation of space policy by commenting on national plans and budgets through our national umbrella technical committees. The technical committees of the AIAA are the organizational units that deal with individual disciplines within the overall structure. The Aerospace Power Systems Technical Committee (APSTC) promotes space power interests through publications, policy support, and the support of the Intersociety Energy Conversion Engineering Conference and other conferences. The level of interaction with electrochemistry is at the user level in the area of space power. While efforts at a more fundamental level are of interest, it is at the lowest individual unit, e.g. the battery cell, solar cell, etc., that our interests become well defined. It is the intent of this paper to describe some of the requirements for the deployment of energy storage technology into operational (nonexperimenta1) power systems.

Even the most advantageous developments in energy storage technology are not assured of adoption for use in space vehicles. Few space programs will adopt new technology in the utility subsystems of a space vehicle unless the risks are well defined and acceptably small, and the actual mission of the program is either enabled by the use of the technology or the penalties in using older, established technologies are severe. Clearly, "better" is insufficient for the adoption of new technology. The success of space efforts when coupled with the conservatism brought on by the tremendous costs of most space operations more often leads to acceptance of reduced performance rather than accepting the risks associated with new technology in the power subsystem. Furthermore, the high reliability and longevity available from current technology sets the standards for future developments. 
The environment for the adoption of new technology is unlikely to change when the penalties for failure are severe. Any technology effort must therefore respond to the conditions and requirements laid down by potential users if it is to be successful. The requirements for adoption can then become a guide to assist in directing research, development, testing, and transition to space applications. These requirements also provide data for estimating the total cost and funding profiles.

The typical energy storage portion of an electrical power system, EPS, for a space vehicle has a reliability requirement, i.e., an estimate of the probability that it will provide for the specifled performance of the mission for the specified duration, greater than 0.97 . After the reliabilities of necessary auxiliary hardware such as cell interconnects, connectors, thermal control features, and mounting fixtures in batteries, and, additionally, pumps, plumbing, and reactant storage in fuel cells are deducted, the rellability requirement for an individual cell unit can exceed 0.9999. Cell reliability requirements can be reduced somewhat if the cell failure mode is a short, or bypass devices protect against open circuit failure without reducing the system reliability; the EPS must also be able to accept lowered and varied delivery voltages from energy storage. Furthermore, the power system and the space vehicle designers really expect more than a specified probability of success. If fallure or wear-out is to occur, the event should be predictable and non-catastrophic. Failures should be soft so that operation can continue at less than specified levels of performance after a component failure. These requirements define both the quantity and quality of the information that must be provided for any space-qualified energy storage device.

The topic of discussion below is the minimum unit development for secondary (rechargeable) battery systems and regenerative fuel cells. This comprises the cell in batteries, but might represent a cell stack in a fuel cell. While these comments also might apply to primary (not recharged) cells, the long life requirements of rechargeable or regenerable units makes development of flight acceptable items both long-term and expensive. It is assumed here that a parallel effort to design and develop the additional requirements for the "integrable" assembly, the battery or the fuel cell unit including tankage, has been performed.

\section{DISCUSSION}

The basic, non-directed research aspects of the development of an energy storage device constitute the smallest portion of the work necessary to bring the technology to fruition. Once a concept is defined and subjected to analysis, a decision to proceed with development can be based on properties that include cost, energy density, electrical characteristics, projected lifetime, development of supporting devices and materials, environmental requirements, safety, and development time. Comparison of new technology with the actual performance of current state-of-the-art cells, as opposed to performance requirements in specifications, will provide an indication of the value of proceeding with the development. Since the real article is being compared with a "projected" technology, truly significant improvements need to be projected. For instance, energy density improvements of fifty percent can rapidly become insignificant as development proceeds. Actual devices are hardly ever as good as projected for a new technology and the stateof-the-art moves forward.

Once a concept has passed muster and proceeds into the various phases of development, it is imperative that preliminary fallure modes and effects analyses 
(FMEA), and reliability analyses be performed. Key elements that impact reliability and longevity should be defined. This is a development tool, not an advertising tool, to demonstrate the level of progress that has been made. The objective is to develop a program to properly define and understand the weak points in the technology. Research programs can then be designed to define the problems and suggest corrective actions as well as the limits of the technology. Often those problems that have the better defined solution receive the most attention while more subtle, long term problems are shunted aside. Directed research on the technology that emphasizes the more subtle and longer term issues should be a continuing effort that extends well into the final, life testing phase of the develop-

ment.

Periodic reviews of the technology to assess its current status in terms of both development and directed research are critical. One must be assured that no practically unsolvable show-stoppers are imbedded in the technology and that the real advantages of the new technology over the state-of-the-art are verified at an early stage. Updating technology comparisons is particularly important because the performance of state-of-the-art technology generally improves while the projections of performance for new technology generally decrease as development moves toward a deliverable product. A realization that the new devices will only be competitive or slightly better than the current state-of-the-art would indicate that the ultimate development will be fruitless and should be cancelled.

Development prototype units permit the validation of the design and confirmation of the FMEA. They should be subjected to straw man qualification testing and abusive environments in order to define the limits of the design(s) and the response of the technology to extrema. These units should be used as guides and not as advertising tools. The intentional or unintentional abusive testing and the need to establish a learning curve for construction and operation of the units limit the value of the operational data acquired from the first units. It is questioned whether preliminary development units should be placed in the data base to establish flight-worthiness. Similarly, poor results at this stage should not bring about cancellation of the program unless the technology is found to be fundamentally flawed. Any difficulties with these cells skews the database with early failures and slows the establishment of adequate reliability estimates. Similarly, the premature testing of any unit in a way that implies that the results are representative of the end item can be very detrimental.

The flight prototype manufacture occurs in the final stages of development and signals the production of a qualified end item. Building on the design, manufacturing, and performance experience, design changes should by now have become an evolutionary rather than a revolutionary process. Future changes should be readily correlated with the data base established with cells produced earlier. The documentation of the manufacturing and testing must be converted from development to production. This generally involves increased definition of requirements, quality monitoring, and a higher level of traceability. An end item FMEA must be generated using the data from both development and research to establish the operational constraints and inherent failure modes of the unit. A detailed performance description should be prepared that describes the performance limits of the item in terms of projected lifetime, electrical capabilities, and environmental constraints. A reliability analysis should be performed to provide assurance that the components have an adequate reliability. However, energy storage devices are limited in reliability by complex chemical and physical interactions within the device and not, typically, by individual component reliability. The reliability and long term performance must be demonstrated before it will be accepted for operational vehicles. 
This is a time consuming and expensive process that requires planning and commitment at the onset of the development program.

The construction and testing of a complete integrable assembly and life testing in some form are required to demonstrate reliability and long term performance. The verification of the integrable unit, the battery or fuel cell assembly, can be by either a ground test or flight test. Environmental testing and proper simulation during life testing generally suffice to demonstrate operation in space for the individual cells. The cell unit is sealed and a space environment should not change the operation of the unit appreciably. Operation cannot depend on gravity or atmospheric pressure; this should have been demonstrated during the preliminary development and design phases. Battery or fuel cell assemblies require experience in integration into spacecraft and demonstration that the new assembly will operate compatibly with its own and other subsystems. Flight testing provides a "warm feeling" but the integration and testing activity is the most valuable portion. Typically, performance data from flight experiments are poorer in quality and limited in range than that acquired on the ground and it is certainly the more expensive to acquire. An additional problem with demonstration flight testing in the future is the trend toward increasing sizes of energy storage flight units. A flight test of a unit sized to provide $5 \mathrm{~kW}$ or more is impractical on most test flights and few programs will risk an entire mission on an unproven energy storage unit. The option then is to flight test a scale model, e.g. 250 to $500 \mathrm{~W}$, or to perform ground tests only.

The requirements of the life testing are to provide long term performance and reliability data, to verify acceptable means of controlling charge and discharge of the units, and to demonstrate an acceptable environmental range for operation. In order to provide statistically significant data, a large number of units must be tested under conditions that adequately simulate actual use. A sufficient number should be subjected to representative qualification level environmental testing to provide assurance that no long term effect exists. The number of units that should be life tested depends on the variations in design and the range of conditions desired. The number of test units can also be traded with the duration of the test; fewer units can be tested for a longer period to demonstrate an arbitrary reliability and confidence level for a specified mission duration. A generic life test of new technology should provide a data base that supports reliability estimates and conditions of operation for appropriate applications. The actual reliability estimates for a specific application will also require mission-specific life testing. Thus a generic life test must have as a goal providing a sufficient data base that will result in establishing an acceptable reliability and confidence level when it is combined with all other available data.

Life testing must be performed within an assured performance envelope. New technology must demonstrate significant performance improvements over existing technology. However, testing new technology at levels near extremities of performance can result in failure of the units and severe delays in the adoption of the technology. A life test should demonstrate the superiority of the technology, but should not risk failure by overtesting. In addition to demonstrating the advantages of the new technology and establishing a data base, another important aspect of a life testing is the promotion of the technology. It should not explore performance limits.

If the technology were to be adopted for a mission at the beginning of the life testing, the probability of success should be quite high, even if relatively undefined. The life test objective is to demonstrate that the existing design(s) are 
highly reliable and acceptable for routine use. The risk in acting on this presumption prior to verification is that long term performance problems will only surface later in the mission. Additionally, the management of the assembly on orbit (charge, discharge, and maintenance) may not have been adequately evaluated. These risks are greatest when the new technology is similar to the existing state-of-theart because the tendency is to assume that the same techniques and procedures can be used on both technologies. A more subtle problem with premature use of the technology is that it will be applied at levels significantly less than its capability in order to mitigate the risk. This then creates a baseline for use that will only slowly change with time (mission experience uber alles) where the technology shows only modest apparent gains over previous technology rather than the level of performance demonstrated in life testing.

As the life testing proceeds successfully and the continuing research further defines the limits of operation and application, the technology can be applied at levels that are nearer the operational limits. Development is then complete and the technology can be used whenever it is required. 